\title{
Variation in the nutrient composition of different feedstuffs for horses
}

\section{Frieder J. Schwarz' , Heike Sliwinski', Manfred Schuster ${ }^{2}$ and Ewald Rosenberger ${ }^{2}$}

Department of Animal Science, Division of Animal Nutrition, Technical University of Munich, Freising-Weihenstephan ${ }^{1}$ and Bavarian State Research Center for Agriculture, Institute of Animal Breeding, Poing ${ }^{2}$

\section{Introduction}

Calculating rations for horses requires the most accurate knowledge possible of the energy and nutrient contents of the individual feedstuffs. In general, average figures from tables of nutritional values (in Germany: DLG-Futterwerttabelle für Pferde, 1995) are used. However, large deviations can be found, leading to possible under- or over-supply of energy or nutrients despite ration planning. This wide ranging investigation of the most common feedstuffs used for horses should provide a general picture of energy and nutrient contents in rations under practical farm conditions.

\section{Material and methods}

Three samples of feedstuffs from forty farms/stables, evenly distributed over Bavaria, were taken, at 4-week intervals during 2001/2002. These individual samples were combined for each of the feedstuff of each stable. The separate ration components investigated were: forage (meadow hay, 1st and 2nd cut, lucerne hay), silages (grass silage, 1 st, 2 nd and subsequent cuts, ryegrass-silage, cereal whole-crop silage, maize silage), grass cobs and maize cobs, cereals (oats, barley, maize) and proprietary horse food/mixes. A total of 159 feedstuffs were analysed, the crude nutrients by the Weende analysis chemically and using the NIRS system. Furtheron, the samples were wet-ashed with acid and then analysed for major and trace elements by AAS. Phosphorus content was determined by colour reaction with an ammoniavanadate/heptamolybdate solution. Digestible crude protein and digestible energy contents were calculated using the analysed crude nutrients and digestibility coefficients for horses from the DLG-Futterwerttabelle (1995).

\section{Results}

In order to get a better general view of the analysed values, only the averages for the conserved grass products (meadow hay, 1 st and 2 nd cut, grass silage, 1 st, 2 nd and subsequent

cuts, grass cobs) and for the cereals (oats, barley, maize) are given, together with the minimum and maximum values showing the range. Table 1 shows the average contents of dry matter, crude nutrients and energy, table 2 the major element contents and table 3 the trace element values.
Table 1 Mean dry matter (DM), crude nutrients (crude ash (CA), crude protein (CP), crude fibre (CF)), digestible crude protein (DP) and digestible energy (DE) of different feedstuffs.

\begin{tabular}{|c|c|c|c|c|c|c|}
\hline & $\begin{array}{c}D M \\
(\mathrm{~g} / \mathrm{kg})\end{array}$ & $\begin{array}{c}C A \\
(\mathrm{~g} / \mathrm{kg} D \mathrm{DM})\end{array}$ & $\begin{array}{c}\mathrm{CP} \\
(\mathrm{g} / \mathrm{kg} \mathrm{DM})\end{array}$ & $\begin{array}{c}\mathrm{CF} \\
(\mathrm{g} / \mathrm{kg} \mathrm{DM})\end{array}$ & $\begin{array}{c}\text { DP } \\
(\mathrm{g} / \mathrm{kg} \mathrm{DM})\end{array}$ & $\begin{array}{c}D E \\
(\mathrm{MJ} / \mathrm{kg} D \mathrm{DM}\end{array}$ \\
\hline $\begin{array}{l}\text { Hay, },^{\text {st }} \text { cut } \\
(\mathrm{n}=43)\end{array}$ & $\begin{array}{c}848 \\
795-24876 \\
\end{array}$ & $\begin{array}{c}60 \\
40-76 \\
\end{array}$ & $\begin{array}{c}78 \\
58-121 \\
\end{array}$ & $\begin{array}{c}331 \\
294-361 \\
\end{array}$ & $\begin{array}{c}46 \\
31-76 \\
\end{array}$ & $\begin{array}{c}8.96 \\
7.6-10.1\end{array}$ \\
\hline \begin{tabular}{|l} 
Hay, $2^{\text {nd }}$ cut \\
$(\mathrm{n}=3)$
\end{tabular} & $\begin{array}{c}826 \\
824-830 \\
\end{array}$ & $\begin{array}{c}68 \\
43-83 \\
\end{array}$ & $\begin{array}{c}104 \\
97-106 \\
\end{array}$ & $\begin{array}{c}295 \\
293-297 \\
\end{array}$ & $\begin{array}{c}64 \\
60-67 \\
\end{array}$ & $\begin{array}{c}9.85 \\
9.7-10.2 \\
\end{array}$ \\
\hline \begin{tabular}{|l|} 
Grass silage \\
$1^{\text {st }}$ cut $(n=12)$
\end{tabular} & $\begin{array}{c}571 \\
420-755 \\
\end{array}$ & $\begin{array}{c}79 \\
53-129 \\
\end{array}$ & $\begin{array}{c}104 \\
62-160 \\
\end{array}$ & $\begin{array}{c}322 \\
268-372 \\
\end{array}$ & $\begin{array}{c}71 \\
42-112 \\
\end{array}$ & $\begin{array}{c}10.0 \\
9.5-11.2 \\
\end{array}$ \\
\hline $\begin{array}{l}\text { Grass silage } \\
2^{\text {nd }} \text { cut }(n=24)\end{array}$ & $\begin{array}{c}540 \\
285-769 \\
\end{array}$ & $\begin{array}{c}130 \\
92-218 \\
\end{array}$ & $\begin{array}{c}123 \\
92-184 \\
\end{array}$ & $\begin{array}{c}259 \\
204-294 \\
\end{array}$ & $\begin{array}{c}89 \\
61-138 \\
\end{array}$ & $\begin{array}{c}9.45 \\
8.2-11.1 \\
\end{array}$ \\
\hline \begin{tabular}{|l} 
Grass cobs \\
$(\mathrm{n}=7)$
\end{tabular} & $\begin{array}{c}906 \\
888-924 \\
\end{array}$ & $\begin{array}{c}127 \\
89-247 \\
\end{array}$ & $\begin{array}{c}114 \\
95-160 \\
\end{array}$ & $\begin{array}{c}241 \\
171-210 \\
\end{array}$ & $\begin{array}{c}97 \\
64-138 \\
\end{array}$ & $\begin{array}{c}9.86 \\
12.5-13.4 \\
\end{array}$ \\
\hline \begin{tabular}{|l|l|} 
Oats \\
$(\mathrm{n}=34)$
\end{tabular} & $\begin{array}{c}870 \\
851-886 \\
\end{array}$ & $\begin{array}{c}29 \\
25-31 \\
\end{array}$ & $\begin{array}{c}115 \\
95-136 \\
\end{array}$ & $\begin{array}{c}116 \\
96-152 \\
\end{array}$ & $\begin{array}{c}91 \\
75-107 \\
\end{array}$ & $\begin{array}{c}13.0 \\
12.5-13.4 \\
\end{array}$ \\
\hline \begin{tabular}{|l|l} 
Barley \\
$(n=10)$
\end{tabular} & $\begin{array}{c}873 \\
859-596 \\
\end{array}$ & $\begin{array}{c}23 \\
22-25 \\
\end{array}$ & $\begin{array}{c}117 \\
98-126 \\
\end{array}$ & $\begin{array}{c}51 \\
49-56 \\
\end{array}$ & $\begin{array}{c}86 \\
72-91 \\
\end{array}$ & $\begin{array}{c}14.5 \\
14.5-14.6 \\
\end{array}$ \\
\hline Maize & $\begin{array}{c}877 \\
861-887\end{array}$ & $\begin{array}{c}16 \\
14-18\end{array}$ & $\begin{array}{c}98 \\
90-108\end{array}$ & $\begin{array}{c}22 \\
19-25\end{array}$ & $\begin{array}{c}68 \\
61-75\end{array}$ & $\begin{array}{c}15.6 \\
15.5-15.6\end{array}$ \\
\hline
\end{tabular}

Table 2 Mean content of major minerals of different feedstuffs.

\begin{tabular}{|c|c|c|c|c|c|}
\hline & $\begin{array}{c}\mathrm{Ca} \\
(\mathrm{g} / \mathrm{kg} \mathrm{DM})\end{array}$ & $\begin{array}{c}P \\
(g / k g ~ D M)\end{array}$ & $\begin{array}{c}\mathrm{Mg} \\
(\mathrm{g} / \mathrm{kg} \mathrm{DM})\end{array}$ & $\begin{array}{c}\mathrm{Na} \\
(\mathrm{g} / \mathrm{kg} \mathrm{DM})\end{array}$ & $\begin{array}{c}\mathrm{K} \\
(\mathrm{g} / \mathrm{kg} \mathrm{DM})\end{array}$ \\
\hline $\begin{array}{l}\text { Hay, } 1^{\text {st }} \text { cut } \\
(\mathrm{n}=43)\end{array}$ & $\begin{array}{c}4.15 \\
1.9-7.2\end{array}$ & $\begin{array}{c}2.25 \\
1.2-3.6\end{array}$ & $\begin{array}{c}1.63 \\
1.0-2.3\end{array}$ & $\begin{array}{c}0.38 \\
0.03-2.0\end{array}$ & $\begin{array}{c}16.9 \\
7.8-33.0\end{array}$ \\
\hline $\begin{array}{l}\text { Hay, } 2^{\text {nd }} \text { cut } \\
(\mathrm{n}=3)\end{array}$ & $\begin{array}{c}5.89 \\
5.0-7.1\end{array}$ & $\begin{array}{c}3.10 \\
2.9-3.5\end{array}$ & $\begin{array}{c}2.07 \\
1.6-2.6\end{array}$ & $\begin{array}{c}0.48 \\
0.05-0.8\end{array}$ & $\begin{array}{c}20.5 \\
10.9-25.7\end{array}$ \\
\hline $\begin{array}{l}\text { Grass silage } \\
1^{\text {st }} \text { cut }(n=12) \\
\end{array}$ & $\begin{array}{c}5.48 \\
2.4-11.6 \\
\end{array}$ & $\begin{array}{c}3.06 \\
2.2-5.0\end{array}$ & $\begin{array}{c}1.91 \\
1.2-3.9 \\
\end{array}$ & $\begin{array}{c}0.20 \\
0.05-0.6\end{array}$ & $\begin{array}{c}25.7 \\
16.1-40.0 \\
\end{array}$ \\
\hline $\begin{array}{l}\text { Grass silage } \\
2^{\text {nd }} \text { cut }(n=24)\end{array}$ & $\begin{array}{c}7.86 \\
3.5-16.9 \\
\end{array}$ & $\begin{array}{c}3.44 \\
2.2-4.9\end{array}$ & $\begin{array}{c}2.75 \\
1.6-4.8 \\
\end{array}$ & $\begin{array}{c}0.29 \\
0.03-2.4 \\
\end{array}$ & $\begin{array}{c}20.9 \\
13.5-33.4 \\
\end{array}$ \\
\hline $\begin{array}{l}\text { Grass cobs } \\
(\mathrm{n}=7)\end{array}$ & $\begin{array}{c}8.01 \\
5.6-11.0 \\
\end{array}$ & $\begin{array}{c}3.22 \\
2.5-3.7 \\
\end{array}$ & $\begin{array}{c}2.85 \\
1.9-3.3 \\
\end{array}$ & $\begin{array}{c}0.50 \\
0.29-1.0\end{array}$ & $\begin{array}{c}21.0 \\
17.6-24.9 \\
\end{array}$ \\
\hline $\begin{array}{l}\text { Oats } \\
(\mathrm{n}=34)\end{array}$ & $\begin{array}{c}0.88 \\
0.61-1.6 \\
\end{array}$ & $\begin{array}{c}3.79 \\
3.4-4.1\end{array}$ & $\begin{array}{c}1.20 \\
1.1-1.5 \\
\end{array}$ & $\begin{array}{c}0.07 \\
0.02-0.3 \\
\end{array}$ & $\begin{array}{c}4.93 \\
4.0-6.8 \\
\end{array}$ \\
\hline \begin{tabular}{|l|} 
Barley \\
$(\mathrm{n}=10)$
\end{tabular} & \begin{tabular}{|c|}
0.53 \\
$0.39-0.69$ \\
\end{tabular} & $\begin{array}{c}3.94 \\
3.6-4.5\end{array}$ & $\begin{array}{c}1.23 \\
1.1-1.3\end{array}$ & $\begin{array}{c}0.07 \\
0.04-0.1\end{array}$ & $\begin{array}{c}5.64 \\
4.5-6.1\end{array}$ \\
\hline $\begin{array}{l}\text { Maize } \\
(n=9)\end{array}$ & $\begin{array}{c}0.20 \\
0.06-0.64\end{array}$ & $\begin{array}{c}3.25 \\
2.9-3.5\end{array}$ & $\begin{array}{c}1.27 \\
1.1-1.4\end{array}$ & $\begin{array}{c}0.07 \\
0.02-0.2\end{array}$ & $\begin{array}{c}3.68 \\
1.4-4.2\end{array}$ \\
\hline
\end{tabular}

Table 3 Mean content of trace elements of different feedstuffs.

\begin{tabular}{|c|c|c|c|c|}
\hline & $\begin{array}{c}\mathrm{Cu} \\
(\mathrm{mg} / \mathrm{kg})\end{array}$ & $\begin{array}{c}M n \\
(m g / k g ~ D M)\end{array}$ & $\begin{array}{c}\mathrm{Zn} \\
(\mathrm{mg} / \mathrm{kg} \mathrm{DM})\end{array}$ & $\begin{array}{c}\mathrm{Se} \\
(\mu \mathrm{g} / \mathrm{kg} D M)\end{array}$ \\
\hline $\begin{array}{l}\text { Hay, } 1^{\text {st }} \text { cut } \\
(n=43)\end{array}$ & $\begin{array}{c}4.60 \\
3.2-6.0\end{array}$ & $\begin{array}{c}97.0 \\
27.3-331 \\
\end{array}$ & $\begin{array}{c}30.9 \\
17.5-44.0\end{array}$ & $\begin{array}{c}23.6 \\
8.2-91.0 \\
\end{array}$ \\
\hline $\begin{array}{l}\text { Hay, } 2^{\text {nd }} \text { cut } \\
(n=3)\end{array}$ & $\begin{array}{c}5.41 \\
5.0-5.8\end{array}$ & $\begin{array}{c}47.5 \\
31.7-64.7 \\
\end{array}$ & $\begin{array}{c}36.4 \\
16.9-47.5 \\
\end{array}$ & $\begin{array}{c}21.0 \\
12.5-36.1 \\
\end{array}$ \\
\hline $\begin{array}{l}\text { Grass silage } \\
1^{\text {st }} \text { cut }(n=12)\end{array}$ & $\begin{array}{c}5.50 \\
3.5-7.7\end{array}$ & $\begin{array}{c}110 \\
36.9-201 \\
\end{array}$ & $\begin{array}{c}31.4 \\
16.9-47.5 \\
\end{array}$ & $\begin{array}{c}19.1 \\
9.3-48.6 \\
\end{array}$ \\
\hline $\begin{array}{l}\text { Grass silage } \\
2^{\text {nd }} \text { cut }(n=24)\end{array}$ & $\begin{array}{c}7.18 \\
6.0-10.4 \\
\end{array}$ & $\begin{array}{c}169 \\
28.6-410 \\
\end{array}$ & $\begin{array}{c}40.9 \\
27.5-73.1 \\
\end{array}$ & $\begin{array}{c}48.4 \\
10.4-197 \\
\end{array}$ \\
\hline $\begin{array}{l}\text { Grass cobs } \\
(n=7)\end{array}$ & $\begin{array}{c}7.89 \\
6.7-10.0 \\
\end{array}$ & $\begin{array}{c}185 \\
100-306 \\
\end{array}$ & $\begin{array}{c}42.3 \\
34.9-54.2 \\
\end{array}$ & $\begin{array}{c}46.1 \\
21.6-81.3 \\
\end{array}$ \\
\hline $\begin{array}{l}\text { Oats } \\
(n=34)\end{array}$ & $\begin{array}{c}3.89 \\
2.5-5.1\end{array}$ & $\begin{array}{c}43.5 \\
17.7-73.2 \\
\end{array}$ & $\begin{array}{c}23.8 \\
15.8-37.6 \\
\end{array}$ & $\begin{array}{c}15.9 \\
6.4-33.4 \\
\end{array}$ \\
\hline $\begin{array}{l}\text { Barley } \\
(\mathrm{n}=10)\end{array}$ & $\begin{array}{c}4.42 \\
2.2-5.5\end{array}$ & $\begin{array}{c}17.6 \\
11.8-24.5 \\
\end{array}$ & $\begin{array}{c}25.8 \\
20.6-33.2 \\
\end{array}$ & $\begin{array}{c}14.4 \\
9.6-21.5 \\
\end{array}$ \\
\hline $\begin{array}{l}\text { Maize } \\
(\mathrm{n}=9)\end{array}$ & $\begin{array}{c}2.58 \\
1.9-3.9\end{array}$ & $\begin{array}{c}6.82 \\
5.2-10.8\end{array}$ & $\begin{array}{c}20.7 \\
17.1-25.7\end{array}$ & $\begin{array}{c}13.8 \\
4.4-40.2\end{array}$ \\
\hline
\end{tabular}

\section{Discussion}

Hay is one of the main constituents in any ration for horses. 43 samples of 1 st cut meadow hay were analysed, and only three of 2 nd cut, showing that hardly any 2 nd cut hay is used.

The relatively high contents of crude fibre (33\% in the DM), coupled with the very low values for crude protein $(8 \%$ in the 
DM) suggest a late harvest date. As rising crude fibre content leads to a reduction in digestibility, the digestible energy value of just under $9.0 \mathrm{MJ} / \mathrm{kg}$ DM is rather low. The average contents of major minerals and trace elements, with the exception of manganese, are also on the low side, and the very large range must be mentioned here.

Grass silage $(n=36)$ is now an important component in rations for horses in many stables, with 2 nd and further cuts often used for silage. However, the DM content of the silages varied enormously between below 30\% and more than $70 \%$, so that we cannot yet say that there are set methods for wilting and ensiling silages for horses. Also, a distinction must be made between silages (DM 45-55\%) with normal fermentation and products with higher DM contents (haylage, DM $>65 \%$ ). Both products require high compression for successful conservation, so that forages in an advanced physiological state are unsuitable. In general, grass silages have higher energy and mineral contents than hay.

Grass cobs are produced by drying the grass using fossil fuels, with subsequent compression into cobs. This process is expensive, requiring a high quality product to justify the expense, but in some cases a high content of ash (25\%) was found, which reduces the nutritional value significantly.

The separate samples of barley and maize varied only little in contents of crude nutrients, energy and minerals. There were, however, big variations in the crude protein and crude fibre contents of oats, therefore leading to a wide range of energy contents. All cereal samples showed low major and trace element contents relative to the requirement of horses.

\section{References}

DLG-Futterwerttabelle für Pferde (1995): Universität Hohenheim, Dokumentationsstelle. 3rd ed., DLG-Verlag, Frankfurt

\author{
F. J. Schwarz \\ Department of Animal Science \\ Division of Animal Nutrition \\ Technical University of Munich \\ 85350 Freising-Weihenstephan \\ Germany \\ schwarzf@wzw.tum.de
}

